\title{
Empleo de herramientas metacognitivas para realizar el proyecto final de licenciatura en la Escuela de Ingeniería ESIME-Culhuacán, del Instituto Politécnico Nacional de México
}

The use of metacognitive tools in the preparation for the end-of-degree dissertation at the School of Engineering ESIME-Culhuacán, the National Polytechnic Institute of Mexico

\author{
Ana María Mendioroz Lacambra ${ }^{1}$ \\ anamaria.mendioroz@unavarra.es \\ Universidad Pública de Navarra, España
}

\section{Resumen:}

Se valora la eficacia de la $\mathrm{V}$ heurística de Gowin y el Mapa Conceptual, para visibilizar el proceso de construcción de conocimiento en tareas investigativas; concretamente para guiar la investigación, concebirla como proceso donde converge la epistemología de la ciencia y la metodología, y para facilitar la comunicación de resultados. Se enmarca en un proyecto de investigación en la Escuela de Ingeniería ESIME, del Instituto Politécnico Nacional de México, con el alumnado del proyecto de titulación de Ingeniería en Computación. Se basa en que son herramientas que desarrollan estrategias metacognitivas, para realizar tareas de investigación. La metodología de corte cualitativo, según el modelo de investigación-acción, facili-

\begin{abstract}
:
This study is aimed at assessing the effectiveness of the use of heuristics $V$ Gowin and Conceptual Map for visualizing the process of building knowledge in research tasks; specifically to guide research, conceive of it as a process where the epistemology of science and methodology converge, and to facilitate communication of results. It is part of a research project at the School of Engineering ESIME in the National Polytechnic Institute of Mexico which lasted for three months and was conducted with students of the Computer Engineering Bachelor Degree. It is based on tools that help develop metacognitive strategies which are essential to conduct research. Qualitative methodology, the model of research action, facilitates data
\end{abstract}

1 Dirección para correspondencia (correspondence address):

Ana María Mendioroz Lacambra. Dpto. de Geografía e Historia. Universidad Pública de Navarra. Campus Arrosadía. 31006 Pamplona (España). 
Empleo de herramientas metacognitivas para realizar el proyecto final de licenciatura en la Escuela de Ingeniería ESIME-Culhuacán, del Instituto Politécnico Nacional de

México

Ana María Mendioroz Lacambra

ta la recopilación de datos mediante un cuestionario que permite evidenciar las creencias del alumnado sobre la investigación, los problemas surgidos durante la misma, el concepto sobre la construcción del conocimiento y la valoración personal sobre las dos herramientas, a nivel de elaboración y en cuanto a su empleo. Para recuperar evidencias, se diseña, a modo de ejemplificación, una plantilla que los estudiantes adaptan a sus necesidades. El análisis de los datos con una orientación cualitativa, permite en la línea de estudios anteriores, poner de manifiesto la efectividad de estas herramientas para realizar tareas investigativas, ya que facilitan la integración de los marcos teóricos y metodológicos, orientan el proceso, su planificación, monitorización y la evaluación de los procedimientos. Ayudan a visibilizar el proceso de construcción de conocimiento favoreciendo la metacognición, e incrementan la seguridad del alumnado, contribuyendo además a mejorar la comunicación de los resultados.

\section{Palabras clave:}

Mapa conceptual; $v$ heurística; investigación; metacognición. collection through a questionnaire, implemented at the beginning and end of the procedure, which makes evident students' beliefs on research, problems encountered during the procedure, the concept of building knowledge and personal assessment of both metacognitive tools, both in terms of development and in terms of their employment. Moreover, to recover evidence of student productions, a template that students adapt to their necessities is designed. The data analysis with a qualitative orientation allows obtaining results consistent with previous studies, reveals the effectiveness of the $V$ of Gowin and Conceptual Map to perform research tasks since this approach facilitates the integration of theoretical and methodological frameworks, guides the process-its planning, monitoring and constant evaluation of the procedures. This approach does also help visualize the process of building knowledge, promoting metacognition, and increases the safety of students, besides helping to improve the communication of results.

\section{Key words:}

Concept mapping; $v$ heuristics; investigation; metacognition.

\section{Résumé :}

On évalue l'efficacité de la $V$ heuristique de Gowin et la Carte Conceptuelle, pour rendre visible le processus de construction de la connaissance dans des tâches d'investigation; concrètement pour guider la recherche, la concevoir comme processus où converge l'épistémologie de la science et de la méthodologie, et pour faciliter la communication de résultats. Il est encadré dans un projet de recherche à l'École d'Ingénierie ESIME, de I'Institut Polytechnique National du Mexique. II se base sur des outils qui développent des stratégies métacognitives, pour réaliser des tâches de recherche. Selon le modèle de recherche - action, la méthodologie qualitative facilite le résumés de données au moyen d'un questionnaire qui permet de mettre en évidence les croyances de l'ensemble des élèves sur la recherche, les problèmes surgis durant la même, le concept sur la construction de la connaissance et de l'évaluation personnelle sur les deux outils, à un niveau d'élaboration et en ce qui concerne son emploi. Pour recueillir des preuves, un modèle est fourni aux étudiants qui l'adaptent à leurs nécessités. L'analyse des données avec une orientation qualitative, permet dans la ligne d'études antérieures, de mettre en évidence l'effectivité de ces outils pour réaliser des tâches d'investigation, puisqu'ils facilitent l'intégration des cadres théoriques et méthodologiques, ils orientent le processus, sa planification, monitorage et l'évaluation des procédures. Ils aident à rendre visible le processus de construction de la connaissance en favorisant la métacognition, et augmentent la sécurité des élèves, en contribuant à améliorer de plus la communication des résultats.

\section{Mots Clés:}

Carte conceptuelle; v heuristique; recherche; métacognition. 
Empleo de herramientas metacognitivas para realizar el proyecto final de licenciatura en la Escuela de Ingeniería ESIME-Culhuacán, del Instituto Politécnico Nacional de

México

ana María Mendioroz Lacambra

Fecha de recepción: 13-4-2015

Fecha de aceptación: 1-2-2016

\section{Introducción}

En el nuevo modelo educativo de Educación Superior, con una dimensión práctica y operativa de la enseñanza, el alumnado se convierte en protagonista de su propio aprendizaje. Con el objeto de que sea capaz de adaptarse a los cambios, convierta la información en conocimiento técnico y humano, tome decisiones, resuelva problemas y en suma, aprenda a aprender con un sentimiento de autoeficacia, debe adquirir competencias instrumentales, interpersonales y sistémicas, imprescindibles para construir explicaciones a partir de la indagación, la experimentación y la contrastación teórica (Roncario, 2012). Investigar exige diseñar y gestionar un proyecto; identificar la fuente del problema, plantear y definir la pregunta que guiará la investigación y valorarla como recurso para profundizar en los conocimientos (Graesser y otros, 2005; Plata, 2011). Además, requiere construir hipótesis de trabajo fundamentadas en un sólido marco teórico y definir con claridad los experimentos, de tal forma que permitan implementar una metodología de trabajo eficaz para obtener datos e interpretarlos y evaluarlos de forma contextualizada y argumentada, desde las teorías aceptadas por la comunidad científica. En suma, el investigador debe ser capaz de integrar la dimensión epistemológica y la metodológica (Muñoz, Quintero y Munévar, 2001), y para ello tiene que desarrollar competencias metacognitivas que posibiliten el empleo de estrategias investigadoras y de procesos de autorregulación de los aprendizajes, de tal forma que le permitan construir explicaciones contextualizadas, proponer soluciones al problema y argumentar, empleando los métodos idóneos (Banthar, 2007; Bruning, Schraw, Norby, y Ronning, 2005; Higuita-López, Molano-Velandia y Rodríguez-Merchán, 2011; Hinton, 2005; Hipkins, 2006; Lin, Schwartz y Hatano, 2005; Prytula, 2008; Sáiz y Román, 2010; Whitebread y otros, 2009).

El empleo de la V heurística de Gowin y del Mapa Conceptual (MC), desarrollan la metacognición y el metaaprendizaje, y su aplicación facilita las tareas investigativas en el aula (Belmonte, 2004; González, 2008; Mendioroz y Guardián, 2014). Ambos recursos, empleados de manera consciente y reflexiva, son herramientas eficaces en la resolu- 
Empleo de herramientas metacognitivas para realizar el proyecto final de licenciatura en la Escuela de Ingeniería ESIME-Culhuacán, del Instituto Politécnico Nacional de México

Ana María Mendioroz Lacambra

ción de problemas (Andres, Meneses y Pesa, 2007; Andrés, Pesa y Moreira, 2006; Flores, Caballero y Moreira, 2011; García, Insausti y Merino, 2003; Mendioroz, 2012; Moreira, 2005; Morales, 1998; Virla 2002; Vizcaya, Asuaje y Gutiérrez, 2009), incluso y más concretamente en el área de conocimiento de Ingeniería en Computación (Guardián, 2009).

\section{Marco Teórico}

El instrumento de la $\mathrm{V}$ heurística ideada por Gowin y desarrollada posteriormente por González y Novak en el marco del constructivismo (González, 1992; Gowin, 1981; Gowin y Álvarez, 2005; Novak y Gowin, 2005), ayuda a comprender la estructura del conocimiento y la forma de construirlo, propiciando el metaconocimiento y el metaaprendizaje. Se basa en cinco preguntas que orientan las fases del método científico, y que apuntan a la pregunta determinante, los conceptos clave, los métodos de investigación, las afirmaciones sobre el conocimiento y los juicios de valor; y permite visualizar la construcción de conocimiento como producto de la investigación, resultado de la interacción entre la estructura conceptual y las metodologías que se emplean para construirlo (Moreira, 2005, 2006).

La forma de $\mathrm{V}$ facilita la permanente interacción entre el lado conceptual/teórico referido al conocimiento disciplinar, y el lado metodológico/ práctico, que se elabora durante el proceso de la investigación. Ambos están dirigidos a los acontecimientos o experimentos necesarios para construir conocimiento, que a su vez necesitan de instrumentos para recopilar las evidencias, transformarlas orientadas por la teoría, y elaborar afirmaciones que respondan a las preguntas e hipótesis que sirven para centrar la investigación.

Por su parte, el Mapa Conceptual es una herramienta muy bien acogida por el alumnado (Reitano y Green, 2013), y facilita la representación visual de la estructura cognitiva de un sujeto. Permite visibilizar cómo ha construido el conocimiento sobre un tema y la manera en la que los esquemas cognitivos se reestructuran, en la medida en la que va introduciendo nuevos conocimientos. Es idónea para conseguir aprendizajes significativos, ya que propicia la reflexión y contribuye a la adquisición de destrezas que favorecen la metacognición y el metaaprendizaje (Cañas y Novak, 2014; González, 2008; Novak y Cañas, 2006; Novak y 
Gowin, 2005). Facilita las tareas investigativas, también en el campo de la ingeniería en computación (Guardián, Veloz, Rodríguez y Veloz, 2013; Veloz, Veloz, Rodríguez y González, 2011a, 2011b), y la comunicación de los resultados, cuando se emplea para la elaboración de textos expositivos (Mendioroz, 2012).

\section{Marco Empírico}

\section{Método}

En este proyecto de orientación básica, se emplea el modelo de investigación-acción (Cohen y Manion, 2002), como una de las formas más conscientes de ejercer la docencia. El propósito de este método de investigación de corte cualitativo, se dirige a que el profesor convertido en investigador, reflexione sobre su práctica educativa de forma que repercuta tanto en la calidad del aprendizaje, como en la propia enseñanza. Esta metodología parte de la indagación sobre la realidad, tomando como origen los problemas prácticos vinculados a la experiencia personal, donde se combinan reflexión y actuación sobre la práctica de aula, con el objeto de mejorarla desde la propia percepción de los sujetos implicados directamente en la acción. En este caso, y ante las dificultades que el alumnado presenta en la elaboración del proyecto de licenciatura, se emplea para valorar el uso de herramientas metacognitivas como facilitadoras del proceso de investigación, al contribuir a que los estudiantes visibilicen la convergencia epistemológica y metodológica de la ciencia, siendo conscientes del proceso de construcción de conocimiento.

\section{Muestra}

Participan en el estudio 10 alumnos, 9 hombres y 1 mujer; 4 de ellos optan por realizar el trabajo de forma personal, y los 6 restantes lo ejecutan de forma colaborativa, en dos grupos de 3 sujetos. Se trata de estudiantes que han obtenido buenas calificaciones a lo largo de la licenciatura, y sin embargo presentan dificultades a la hora de acometer las tareas encargadas por los asesores del proyecto. En algunos casos, incluso tienen problemas para redactar el artículo final que se les encarga. Todos los participantes construyen la $V$ de Gowin, mientras que el MC lo 
Empleo de herramientas metacognitivas para realizar el proyecto final de licenciatura en la Escuela de Ingeniería ESIME-Culhuacán, del Instituto Politécnico Nacional de México

Ana María Mendioroz Lacambra

acometen aquellos que presentan problemas en la comunicación de los resultados, un alumno lo realiza de forma individual y tres de manera colaborativa.

\section{Instrumento}

El instrumento diseñado para la recopilación de los datos (Tabla 1), se basa en un cuestionario de preguntas abiertas que se implementa antes y después de la instrucción en ambos recursos. Su objetivo es recabar información referente a las creencias que el alumnado ha desarrollado sobre la investigación y la forma en la que se construye el conocimiento, así como sobre los problemas surgidos durante la misma y la valoración personal de las dos herramientas metacognitivas, tanto a nivel de elaboración como en cuanto a su empleo. Para construir estas cuestiones y poder contextualizar los datos, se han tenido en cuenta las planteadas en estudios anteriores a propósito de las creencias del alumnado sobre los MC (Pontes, 2014; Pontes, Serrano y Muñoz, 2015). Además, para recopilar las producciones del alumnado referentes a la $\mathrm{V}$, se ha diseñado una plantilla de la herramienta con los principales conceptos que intervienen en la investigación (Figura 1), y otra como guía para volcar el conocimiento necesario para realizar el proyecto, teniendo en cuenta los contextos y enfoques concretos (Figura 2). Finalmente, se les entrega un mapa con los contenidos de la $\mathrm{V}$, con el objeto de fijar lo aprendido sobre esta herramienta y ejemplificar la elaboración del MC que deben construir para organizar el conocimiento y transformarlo en texto (Figura $3)$.

\section{Tabla 1. Encuesta de preguntas abiertas. Elaboración Propia.}

1. Qué dificultades has tenido durante la licenciatura

2. Qué es para ti investigar

3. Cómo se construye el conocimiento en ingeniería en computación

4. Qué problemas estás teniendo durante el diseño y desarrollo de tu proyecto de licenciatura

5. Qué problemas has tenido para redactar el artículo

6. Principales dificultades para elaborar la $\mathrm{V}$

7. Qué te ha aportado y cómo te has sentido al realizarla y emplearla

8. Principales dificultades para elaborar el MC;

9. Qué te ha aportado y cómo te has sentido al realizarlo y emplearlo 


\section{Procedimiento}

\section{$1^{\mathrm{a}}$ Fase}

Se propone la formación en estas dos herramientas a todo el alumnado que cursa los estudios de Proyecto de Licenciatura, con el objeto de mejorar la capacidad de gestión del mismo y facilitar la elaboración del artículo de investigación. Acceden a colaborar finalmente 10 alumnos, aquellos que han presentado dificultades para avanzar en alguna tarea encargada por los asesores. Se inicia la investigación con la implementación de las cinco primeras preguntas del cuestionario, que son respondidas de manera personal.

\section{$2^{\text {a }}$ Fase}

La siguiente fase es la de instrucción. El objetivo es que el alumnado comprenda la estructura del conocimiento y su proceso de construcción, con la intención de que sea capaz de monitorizar su trabajo. La secuencia formativa se inicia con dos sesiones teóricas de $2 \mathrm{~h}$, en las que se imparte instrucción teórica sobre la herramienta heurística denominada V de Gowin. Una vez aclaradas las dudas, se les proporciona un esquema (Figura 1) donde se exponen los principales conceptos que intervienen en la investigación y sus interrelaciones. Se completa la formación con otra plantilla que sirve para volcar el conocimiento necesario en la realización de cada proyecto, teniendo en cuenta los contextos y enfoques concretos (Figura 2).

TEORIA

PRÁCTICA

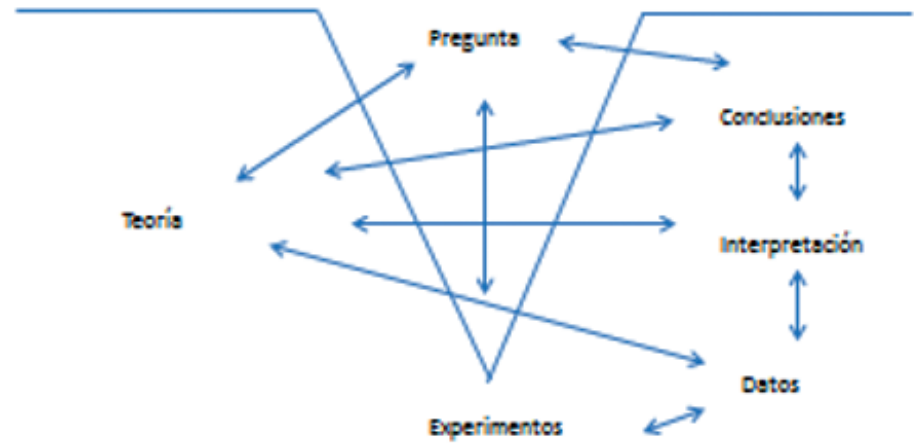

Figura 1. Esquema V de Gowin. Principales conceptos que intervienen en la investigación. Elaboración propia. 
Empleo de herramientas metacognitivas para realizar el proyecto final de licenciatura en la Escuela de Ingeniería ESIME-Culhuacán, del Instituto Politécnico Nacional de México

Ana María Mendioroz Lacambra

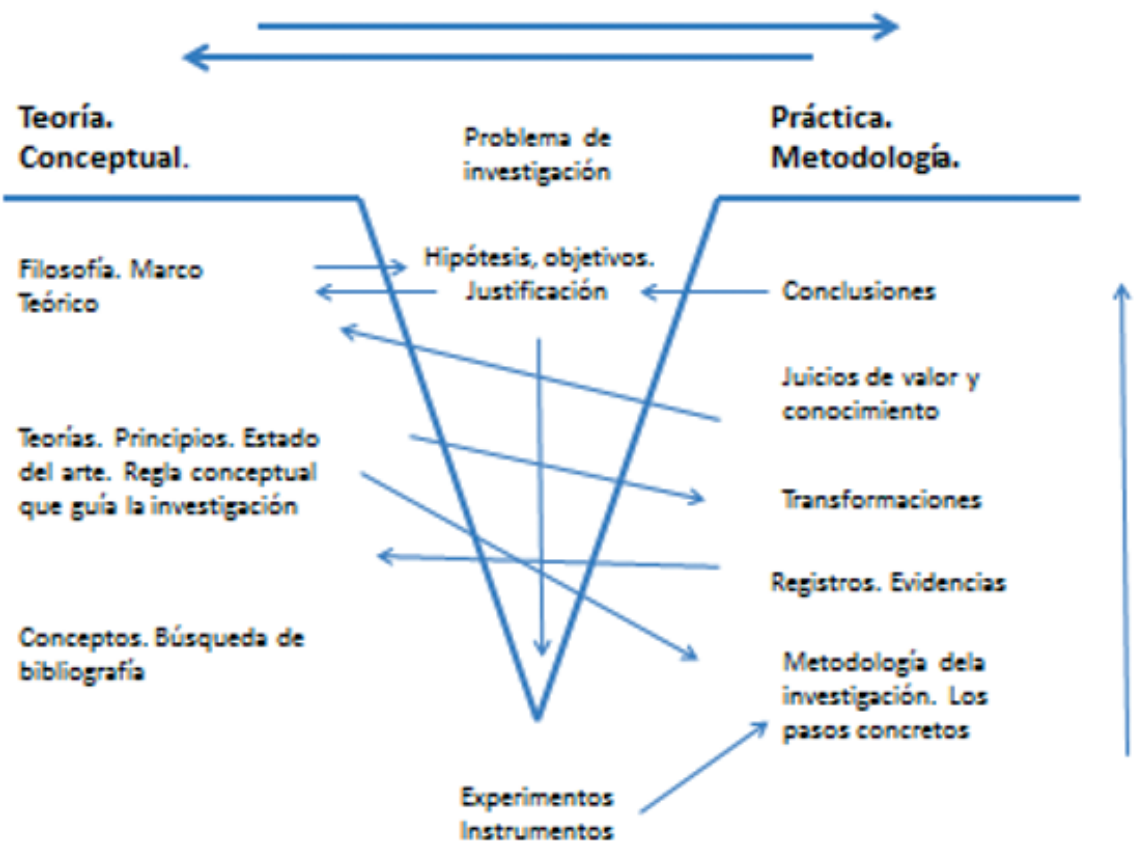

Figura 2. Esquema V de Gowin. Contextos y enfoques. Elaboración propia.

Durante otras dos sesiones de 2 horas cada una, se les instruye en el concepto y elaboración del MC. Para consolidar el procedimiento y fijar lo aprendido sobre la $V$ de Gowin, se les entrega además un MC sobre los contenidos de esta herramienta, que además es útil para reflexionar sobre el proceso de construcción del conocimiento, y por tanto para desarrollar la metacognición (Figura 3). 
Empleo de herramientas metacognitivas para realizar el proyecto final de licenciatura en la Escuela de Ingeniería ESIME-Culhuacán, del Instituto Politécnico Nacional de

México

ana María Mendioroz Lacambra

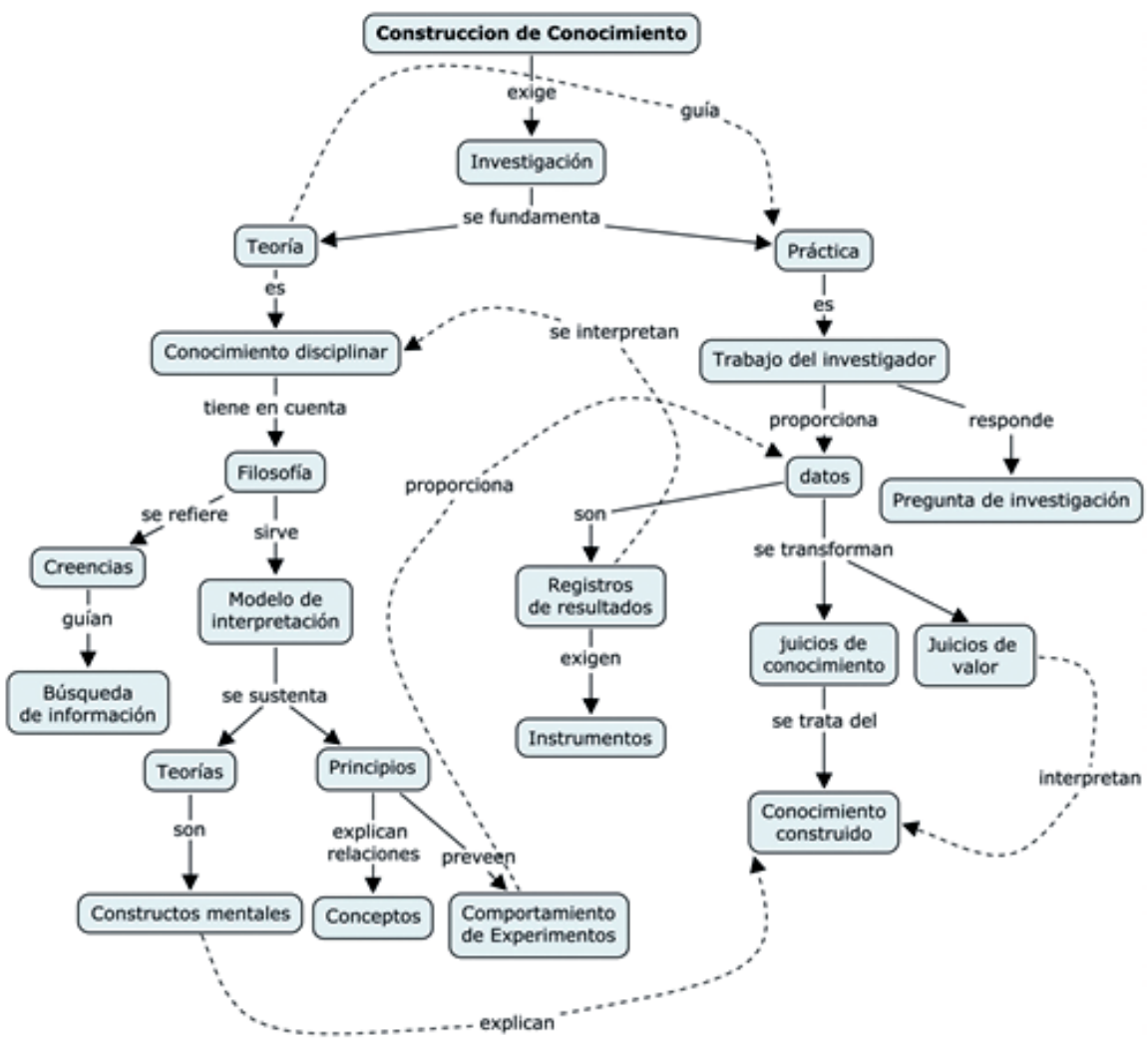

Figura 3. Mapa Conceptual con la teoría sobre la V de Gowin. Elaboración propia.

\section{$3^{\mathrm{a}}$ Fase}

Una vez que los sujetos han sido instruidos en estos recursos propiciadores y facilitadores del desarrollo metacognitivo, se les solicita que transfieran su conocimiento y apliquen lo aprendido. Deben elaborar una $\mathrm{V}$, teniendo en cuenta todos los elementos de la herramienta, adaptada a cada proyecto. Una vez realizada, se les solicita que analicen los pasos reales que han dado a lo largo del semestre, y que de forma reflexiva los evalúen, detecten los posibles errores cometidos y replanteen el proceso, aplicando los ajustes pertinentes. Esta fase es apoyada por la profesora, con tutorías tanto presenciales como telemáticas. Por último, se les solicita que valoren la herramienta y respondan las preguntas seis y siete del cuestionario. 
Empleo de herramientas metacognitivas para realizar el proyecto final de licenciatura en la Escuela de Ingeniería ESIME-Culhuacán, del Instituto Politécnico Nacional de México

ana María Mendioroz Lacambra

En referencia al $\mathrm{MC}$, se inicia el procedimiento con una explicación sobre los contenidos que deben figurar en el resumen de su trabajo, tarea encomendada por los asesores para publicar un artículo. Reflexionan sobre la importancia de la comunicación de los resultados en tareas investigativas, y analizan la pertinencia de los núcleos conceptuales empleados. Finalmente, elaboran un MC teniendo en cuenta lo aprendido sobre la herramienta. Deben seleccionar, jerarquizar e interrelacionar los conceptos y reflexionar sobre el proceso de construcción del conocimiento. Una vez corregidas las producciones, tanto de forma individual como grupal, se les solicita que transformen el MC en texto y lo comparen con el elaborado antes de iniciar el procedimiento. Como en el caso del recurso anterior, deben valorar la herramienta y responder las dos últimas preguntas del cuestionario.

\section{Resultados y discusión}

Para presentar las evidencias recuperadas, y teniendo en cuenta la limitación de espacio, se ha optado por incluir todas las producciones del alumnado referentes a ambas herramientas, ofrecer un resumen de las ideas principales respecto a las dos primeras preguntas, y transcribir las opiniones volcadas en el cuestionario del resto de las cuestiones, indicando entre paréntesis el número de sujetos que han respondido de forma similar. El análisis de los datos se ha realizado intra-sujeto.

Con respecto a la primera pregunta qué dificultades has tenido durante la licenciatura, el alumnado coincide en responder que no ha tenido problemas relevantes, incluso en que ha cursado sus estudios con buenas calificaciones.

Sobre las ideas que han desarrollado respecto a la investigación, que se refiere a la segunda cuestión qué es para ti investigar, las respuestas en todos los casos enfatizan la realización de "experimentos", con el objetivo de innovar en el campo de la ingeniería en computación.

Cuando se les ha interrogado acerca de cómo se construye el conocimiento en ingeniería en computación, 4 alumnos dan preeminencia a la actividad experimental, 3 priman los fundamentos teóricos, 2 mencionan la investigación y 1 las revistas y congresos. Como se observa por las evidencias recuperadas, no son capaces de visibilizar el proceso de construcción de conocimiento, ni la investigación como una 
convergencia entre teoría y práctica, demostrando muy bajo dominio en competencias investigadoras. Las respuestas están en la línea de las siguientes: "La parte fuerte es la teórica, pues se deben integrar todos los fundamentos teóricos en una interfaz de control (1alumno); con una actividad experimental (3); es el estado del arte (2); mediante experimentos teniendo en cuenta el número de medidas y las variables (1); son útiles los congresos y las revistas donde se divulgan los avances(1); como en las demás disciplinas investigando(2)"

En la cuarta pregunta, qué problemas estás teniendo durante el diseño y desarrollo de tu proyecto de licenciatura, 6 alumnos manifiestan haber tenido serias dificultades para concretar la pregunta guía de la tesis, 3 declaran haber primado la parte teórica y 4 la parte práctica, pero en ningún caso son capaces de vincularlas. Hasta en 9 ocasiones manifiestan dificultades para desarrollar el proceso investigativo, en 3 apuntan hacia el desconocimiento de los procedimientos, y finalmente 6 alumnos se sientes decepcionados. Son conscientes de la importancia que el marco teórico tiene en las tareas investigadoras, pero no son capaces de integrar el marco teórico y metodológico, hasta el punto de que no evidencian su convergencia y lo que ello implica. Desconocen el proceso real de construcción de conocimiento, reconociendo que la investigación ha sido dirigida por el asesor, limitando su papel a la realización de tareas de forma mecánica. La falta de formación en procedimientos investigativos adecuados y eficaces, genera no sólo pérdida de tiempo, sino también sentimientos de fracaso y frustración. En suma, no son autónomos al carecer de las herramientas y las competencias necesarias para realizar tareas de investigación, influyendo no sólo en la eficacia sino también en la autoestima. Las respuestas están en la línea de las siguientes: "fue difícil iniciar la trayectoria, porque aunque sabíamos qué nos interesaba investigar, nos costó definirlo(6); nosotros comenzamos por el marco teórico pero luego no sabíamos comprobar si lo que íbamos realizando era conveniente (1); la fase experimental ocupó buena parte del tiempo(4); yo no entiendo el proceso, es complicado seguir adelante (4); invertimos mucho tiempo pero no sabemos si lo estamos realizando correctamente (1); hubiera interesado conocer cómo lo hacen los que saben hacerlo(2); las teorías son las que explican los experimentos, pero desconozco cómo se comprueba(2); hemos tenido problemas a la hora de ponerlo en práctica(1); yo pensaba que no iba a tener problemas, pero estoy decepcionado(6); se nos indica qué hacer 
Empleo de herramientas metacognitivas para realizar el proyecto final de licenciatura en la Escuela de Ingeniería ESIME-Culhuacán, del Instituto Politécnico Nacional de México

ana María Mendioroz Lacambra

y cómo y lo hacemos de forma mecánica(3); desconozco si se podría haber realizado de otra forma o teniendo en cuenta otros enfoques(1)"

En cuanto a la redacción del artículo, las dificultades que manifiesta el alumnado tienen que ver fundamentalmente con la selección y organización de los contenidos, poniendo en evidencia una vez más, el bajo desarrollo alcanzado en competencias metacognitivas, imprescindibles para comprender el proceso de construcción de conocimiento. Las respuestas fueron las siguientes: "me falta un campo de visión amplio sobre la tesis y es difícil diferenciar los rasgos que den coherencia; desconozco cómo ubicar los conceptos más importantes que den fondo de contenido a los diferentes aspectos a tratar; tenemos muchos datos en bruto y es muy dificultoso entresacar lo que debe ir en cada apartado del artículo; me es difícil ordenar y organizar todos los datos que son muchos".

En lo que se refiere a las creencias desarrolladas por el alumno en cuanto a la elaboración y empleo de ambas herramientas, así como a las sensaciones y sentimientos experimentados durante el proceso, están en sintonía con estudios precedentes en el caso de los mapas conceptuales (Pontes, 2014; Pontes, Serrano y Muñoz, 2015), ya que en un elevado porcentaje de los casos, la asumen como una técnica fácil de aprender, de hecho 3 de los 4 alumnos así la valoran, aunque requiera de mucha ejercitación, tal y como apostillan en los 4 casos. La mayor dificultad es la selección y organización de las ideas principales, así como la elección de las palabras de enlace, tal y como apuntan 3 alumnos. En todos los casos, indican que la herramienta les ha servido para reflexionar, organizar y jerarquizar las ideas, así como para comunicar los resultados de manera eficaz. La elaboración del MC, facilita la selección, jerarquización e interrelación de los conceptos relevantes para construir el resumen del artículo; además, su transformación en texto, no sólo simplifica la redacción del mismo, sino que además hace visible la forma de construir el conocimiento en su área específica. Finalmente, todos responden que se han sentido muy bien aprendiendo la técnica. Las evidencias recuperadas son las siguientes: "ver cómo unas ideas son susceptibles de ser integradas en otras; obtener un campo de visión más amplio acerca del desarrollo de la tesis; ubicar aquellos conceptos más relevantes que dieran fondo de contenido a los diferentes aspectos a tratar durante la escritura de la sección; no sale bien en el primer intento; ayuda a percibir de forma más sencilla la dirección a tomar durante su traducción al documento escrito; puedo decir que los mc son una herramienta que tiene que ser necesaria durante 
Empleo de herramientas metacognitivas para realizar el proyecto final de licenciatura en la Escuela de Ingeniería ESIME-Culhuacán, del Instituto Politécnico Nacional de

México

Ana María Mendioroz Lacambra

el análisis y desarrollo de cualquier tema de investigación, debido a que son de gran ayuda y permiten observar rasgos de análisis de información que en ocasiones nos es difícil diferenciar o dar coherencia; el problema que teníamos inicialmente era generar datos en bruto acerca de nuestro proyecto por lo que se nos hacía difícil jerarquizar y ordenar los conceptos que íbamos a utilizar; con la realización de este mapa podemos plantear los niveles de importancia que existen en un tema; nuestra definición de mc era muy errónea, ya que seguíamos texto lineal; permite saber qué sabes y en mayor medida cómo lo has aprendido lo que te ayuda a acomodarlo; nos ayuda a acomodar nuestras ideas para así poder plasmarlas posteriormente de forma coherente y organizada".

Presentamos en primer lugar el texto redactado de forma individual por un alumno antes de la instrucción en la herramienta, a continuación el MC elaborado por el sujeto (Figura 4) y finalmente, el resultado de su conversión en texto.

"En este trabajo se propone el análisis, codificación de dos algoritmos que posibiliten la inserción de marca de agua tanto en computadoras como una unidad de procesamiento gráfico y en computadoras de uso común con una unidad de procesamiento centralizado. Serán implementados los algoritmos de inserción aditiva y multiplicativa los cuales dentro de las categorías del dominio de inserción se encuentran en el dominio espacial. Las técnicas de dominio espacial son las primeras que se estudiaron e implementaron y se basan en esquemas relativamente simples. Una técnica que trabaja en el dominio espacial inserta la marca de agua modificando directamente el valor de determinados pixeles de los cuadros de video y donde las reglas de inserción determinan las localizaciones de los pixeles a ser modificados y la fuerza de inserción de la marca de agua"

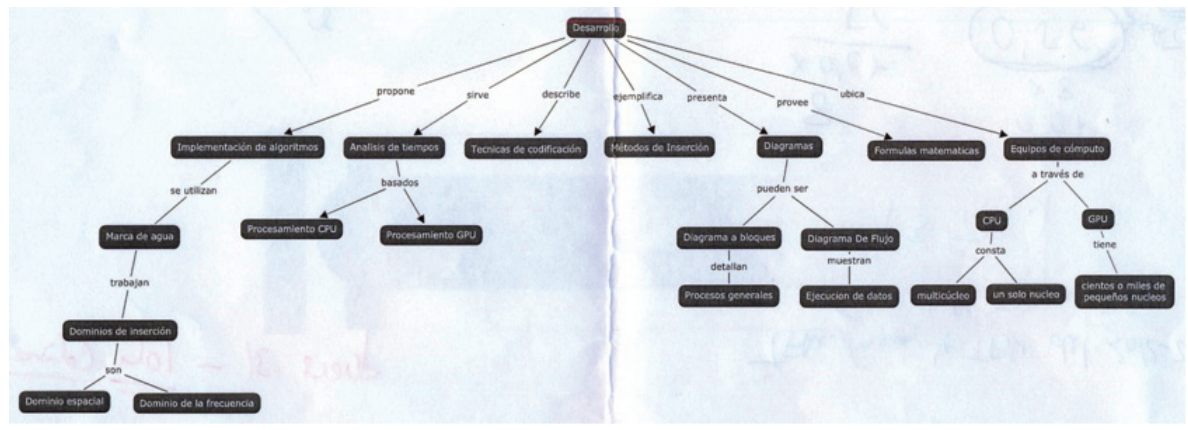

Figura 4. Mapa Conceptual elaborado por el alumno. 
Empleo de herramientas metacognitivas para realizar el proyecto final de licenciatura en la Escuela de Ingeniería ESIME-Culhuacán, del Instituto Politécnico Nacional de México

Ana María Mendioroz Lacambra

"En este apartado se propone la implementación de algoritmos que se utilizan para la inserción de marcas de agua, que pueden trabajar en dominios de inserción diferentes: espacial y de la frecuencia. Sirve para el análisis de tiempos basados en el procesamiento en CPU y en GPU. Describe las técnicas de codificación y ejemplifica los métodos de inserción; además de presentar los diagramas de bloques, para detallar procesos generales, y de flujo, para mostrar la ejecución de los datos, provee de fórmulas matemáticas que establecen los algoritmos de inserción aditiva y multiplicativa para poder ubicar los equipos de cómputo a través de la CPU, con un solo núcleo o varios, y la GPU, con cientos o miles de pequeños núcleos de procesamiento."

Seguidamente, y de la misma forma que en el caso anterior, presentamos en primer lugar el texto redactado en esta ocasión de forma grupal antes de la instrucción en la herramienta, seguido del mapa conceptual elaborado de forma colaborativa (Figura 5) y posteriormente, el resultado de su conversión en texto.

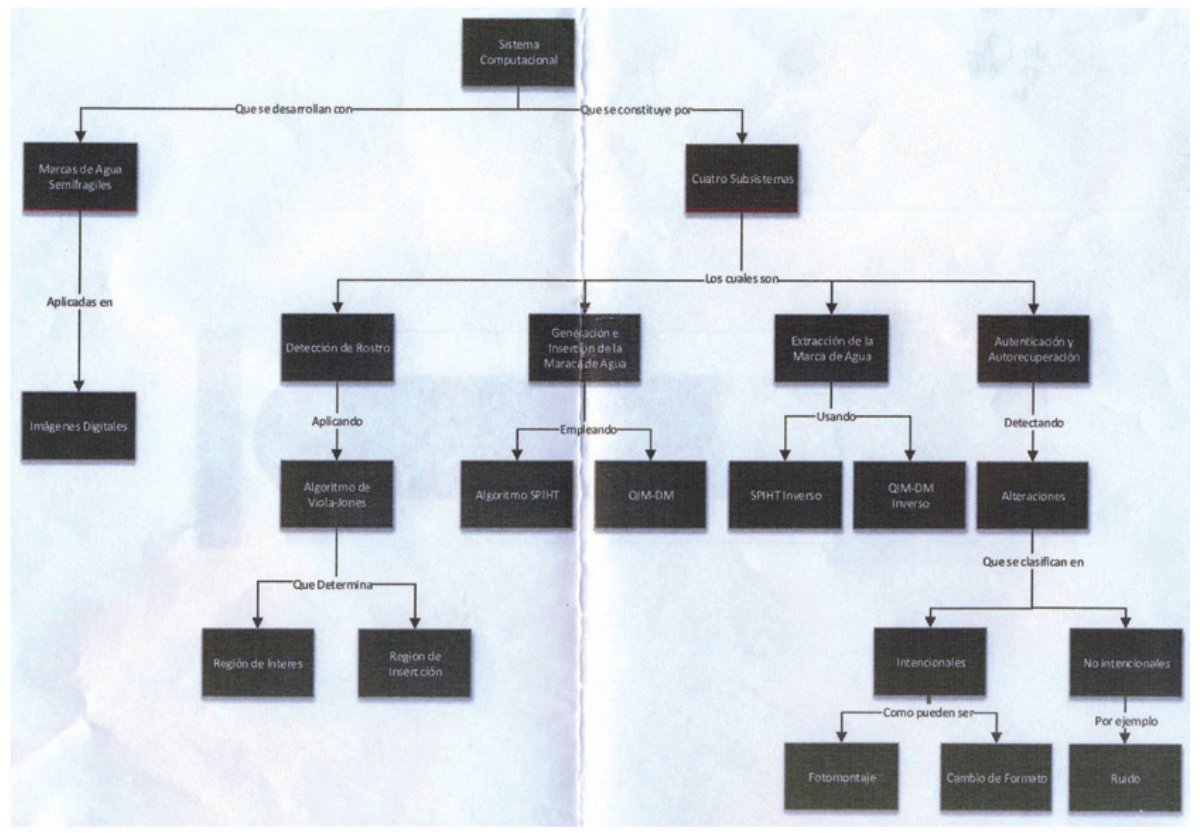

Figura 5. Mapa Conceptual elaborado de forma grupal.

"El sistema tiene como objeto I proteger un área de interés, en este caso es el rostro humano que pueda estar presente en una imagen digital, 
para esto se utiliza el algoritmo de detección de rostros de Viola Jones, Una vez obtenida la información acerca del área de interés y la de inserción, es necesario representar dicha información en el sistema binario y comprimirla para poder insertar la menor cantidad representativa posible. Estos datos son procesados por el algoritmo de compresión SPIHT"

"El sistema computacional que se desarrolla en base a marcas de agua semi-frágiles aplicadas a imágenes digitales, se encuentra constituido por cuatro sub-sistemas. El primero es la detección de rostro en el que se aplica el algoritmo de Viola Jones, que determina la región de interés y la de inserción. El segundo consiste en la generación e inserción de la marca de agua empleando el algoritmo de compresión SIPHT y la técnica de inserción QIM-DM. En el tercer subsistema, se extrae la marca de agua empleando los algoritmos inversos de QIM-DM y SPIHT respectivamente. Por último, el subsistema encargado de la autenticación y auto-recuperación de la región de interés, capaz de detectar las alteraciones tanto intencionales como son fotomontajes y cambio de formato, como no intencionadas, el ruido, para comprobar la robustez del sistema".

Concluimos la exposición y análisis de resultados con las ideas desarrolladas por el alumnado sobre la $\mathrm{V}$ de Gowin, y a pesar de que no podemos compararlas con estudios precedentes y esta muestra no permite la extrapolación de los resultados, las evidencias recuperadas indican que se trata de una herramienta con mayor nivel de complejidad a la hora de integrar todos sus elementos, y sin embargo su aplicación requiere menos ensayos, así lo piensan los 4 alumnos que han construido ambas. Todas las evidencias apuntan a que el recurso sirve como guía para la investigación. Permite integrar los aspectos teóricos y metodológicos como un proceso convergente y evidenciar la forma en la que se construye el conocimiento. Ha favorecido el desarrollo de competencias metacognitivas, imprescindibles para desarrollar tareas investigativas que requieren de la planificación, monitorización y evaluación del proceso. Son conscientes de que las herramientas para iniciar el proceso no habían sido las idóneas y se sintieron fortalecidos y con capacidad de arriesgar intelectualmente, al emplear el recurso. Algunas de las respuestas fueron las siguientes: "En nuestro proyecto se ha enfatizado la parte práctica, y no se ha revisado cada punto práctico con la parte teórica; me ha ayudado a plantear de mejor manera las relaciones entre la teoría y la práctica; nosotros comenzamos por el marco teórico para poder puntualizar nuestra información y la base del proyecto, pero ahora nos 
Empleo de herramientas metacognitivas para realizar el proyecto final de licenciatura en la Escuela de Ingeniería ESIME-Culhuacán, del Instituto Politécnico Nacional de México

ana María Mendioroz Lacambra

damos cuenta que no hemos realizado las comprobaciones pertinentes entre nuestros conocimientos y los resultados; al comienzo me centré en la parte práctica, dejando un poco de lado la parte teórica y ahora me doy cuenta que es bastante complicado seguir con un proyecto cuando no se tienen en cuenta los conocimientos técnicos específicos que desarrolla la teoría; pienso que seguramente habría sido más sencillo iniciar el proyecto por la teoría y toda la actividad experimental ir vinculándola constantemente con el marco teórico; yo comencé por el estado del arte ya que había visto proyectos con anterioridad, pero me costó buscar bibliografía ahora veo cómo hacerlo; en nuestro proyecto no hemos apoyado cada logro experimental con una teoría posible; pensé que lo único importante era el estado del arte y cada disciplina tiene su propia metodología y ahora lo veo más claro; hemos perdido mucho tiempo sin saber si lo que hacíamos estaba bien; experimentar no es sólo valorar el número de medidas y las variables; la contrastación experimental requiere de planificación y control; con la $V$ he visto que los métodos empleados son influenciados por las ideas y teorías que el investigador posee, y viceversa; se logra diferenciar las fases y que está interrelacionado el experimento con la teoría; al diagramar las fases y relacionarlas se ve realmente todo el trabajo es importante evaluar cada paso; aunque es compleja, una vez que se ha comprendido, se lleva a la práctica más rápidamente que el mapa conceptual"."

En las siguientes evidencias, se constata el diferente grado de construcción de conocimiento realizado por el alumnado.

Como se puede observar en la primera imagen, (Figura 6) realizada por el alumno 1, el estudiante ha integrado los aspectos teóricos y metodológicos, teniendo en cuenta el contexto de su proyecto. Transforma el lado de la teoría a partir de la plantilla entregada por la profesora, en marco teórico, estado del arte, leyes y conceptos. Plantea la propuesta de investigación en la parte central, e incluye la hipótesis y la justificación. Es consciente de que la pregunta inicial de la investigación se contesta mediante acciones o experimentos que deben estar dirigidos desde la interacción de la teoría y la metodología apropiada. 
Empleo de herramientas metacognitivas para realizar el proyecto final de licenciatura en la Escuela de Ingeniería ESIME-Culhuacán, del Instituto Politécnico Nacional de

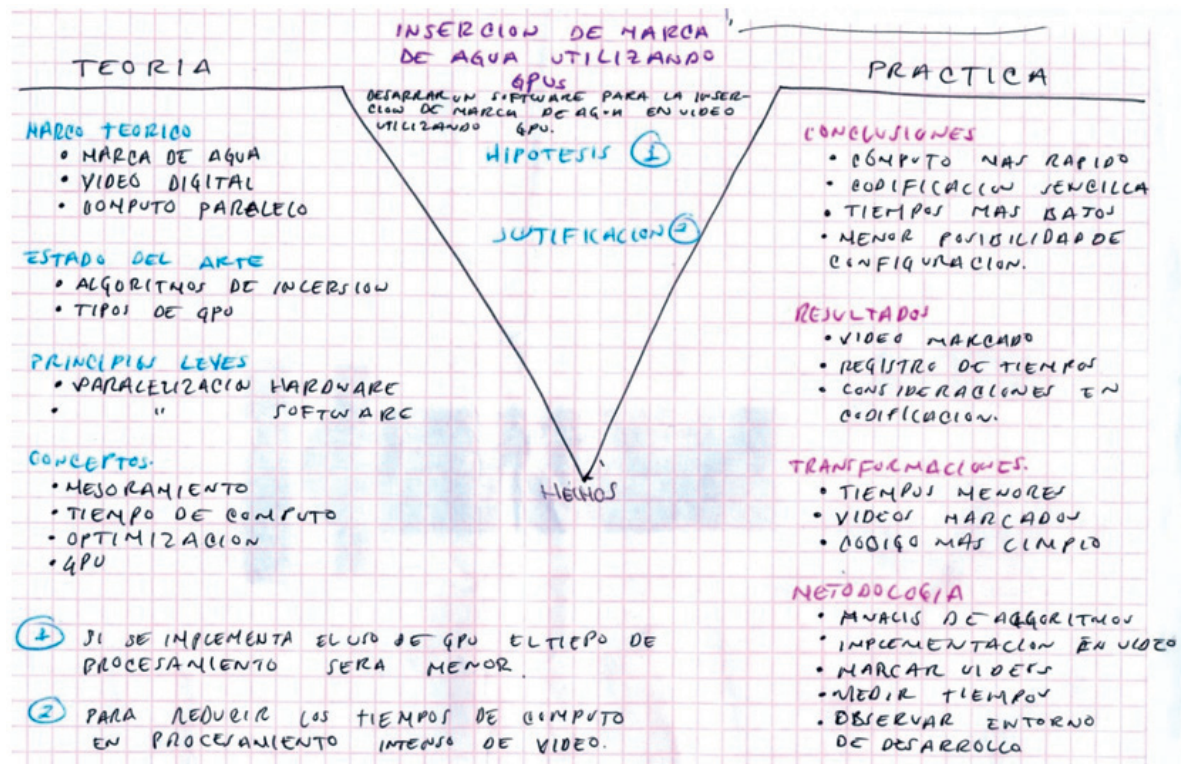

Figura 6. V de Gowin. Elaborada por alumno 1.

En la segunda imagen, (Figura 7) realizada por el alumno 2, se aprecia la integración de los aspectos teóricos y metodológicos, teniendo en cuenta el contexto de su proyecto. Acomete la teoría como en el caso anterior y plantea la propuesta de investigación a modo de pregunta. En la parte central, además de la hipótesis introduce los objetivos y es consciente de que la pregunta inicial se contesta mediante acciones o experimentos que deben estar dirigidos desde la interacción de la teoría y la metodología apropiada, indicándolo mediante flechas que marcan la direccionalidad de las acciones. El empleo del recurso le ha permitido evidenciar el proceso real de construcción de conocimiento. 
Empleo de herramientas metacognitivas para realizar el proyecto final de licenciatura en la Escuela de Ingeniería ESIME-Culhuacán, del Instituto Politécnico Nacional de México

Ana María Mendioroz Lacambra

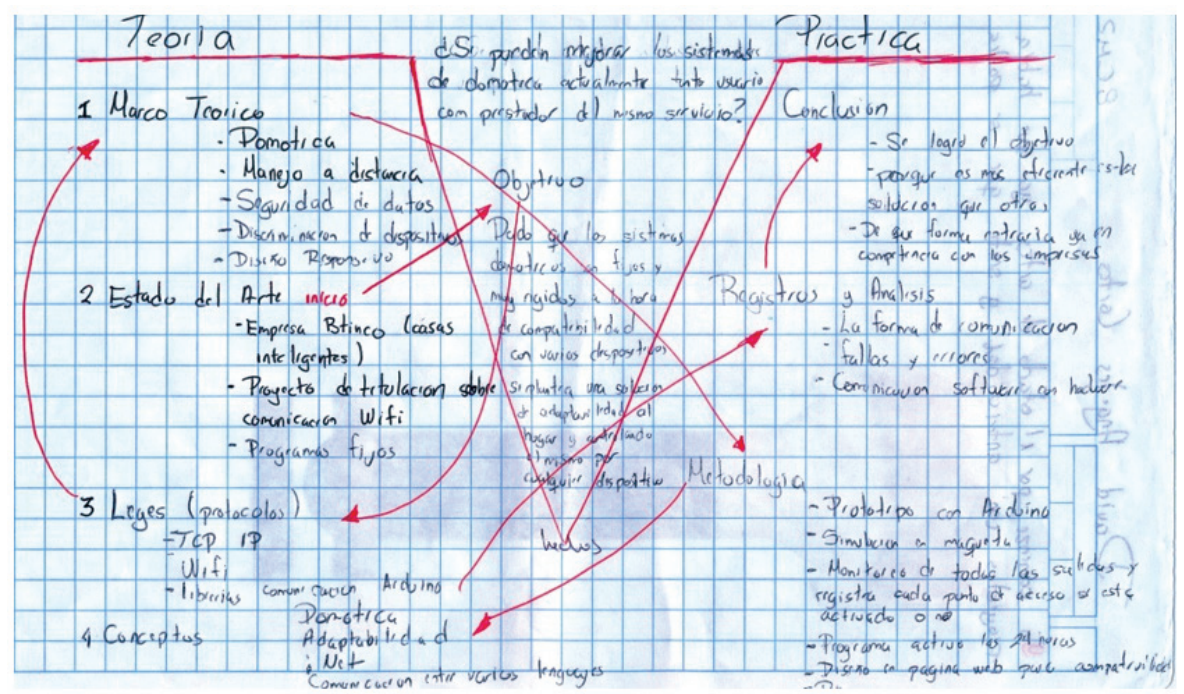

Figura 7. V de Gowin. Elaborada por alumno 2.

En la Figura 8, realizada por el grupo1, se introduce la hipótesis en la parte de la teoría, y se adapta ésta al contexto del proyecto. La doble direccionalidad de las flechas, indica que se ha tenido en cuenta la necesidad de un enfoque teórico-práctico para verificar los resultados del proyecto de investigación.

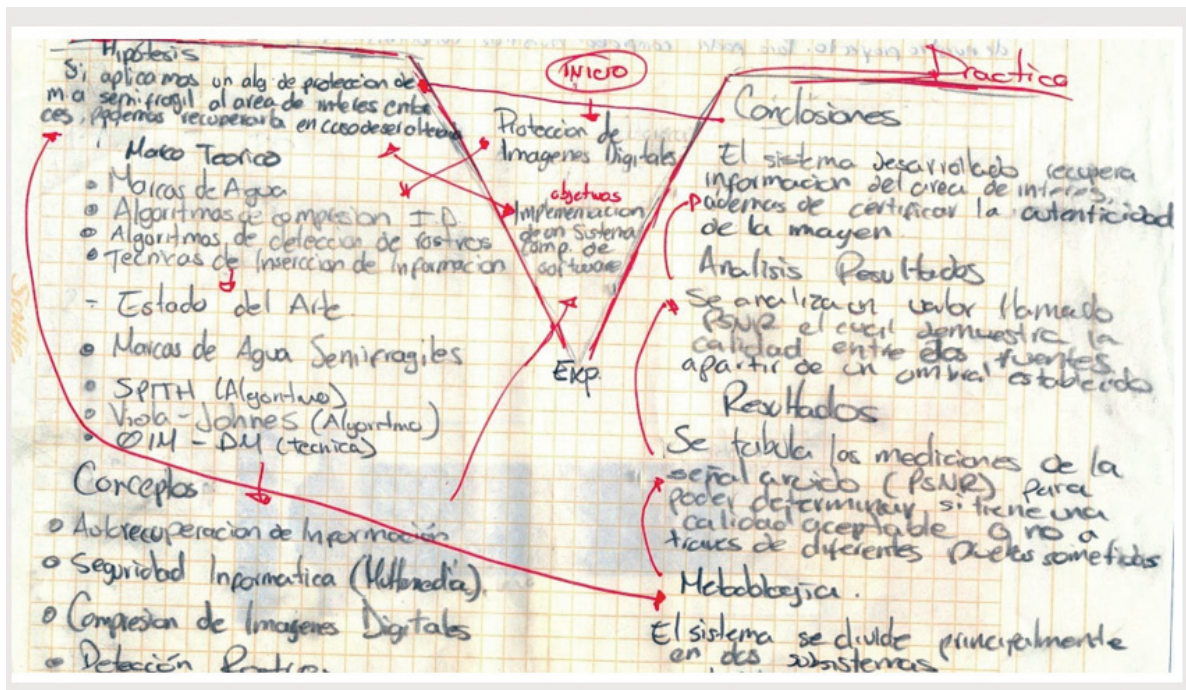

Figura 8. V de Gowin. Elaborada por grupo 1. 
En la Figura 9, el estudiante 3 introduce la justificación en la parte central y dirige la pregunta hacia los experimentos. Emplea en la parte teórica los mismos conceptos propuestos por la profesora, y aunque cambia la direccionalidad de los términos de la parte práctica, y no integra las acciones entre ambas mediante flechas, en la reflexión se constata que es capaz de reconocer la convergencia entre teoría y práctica.

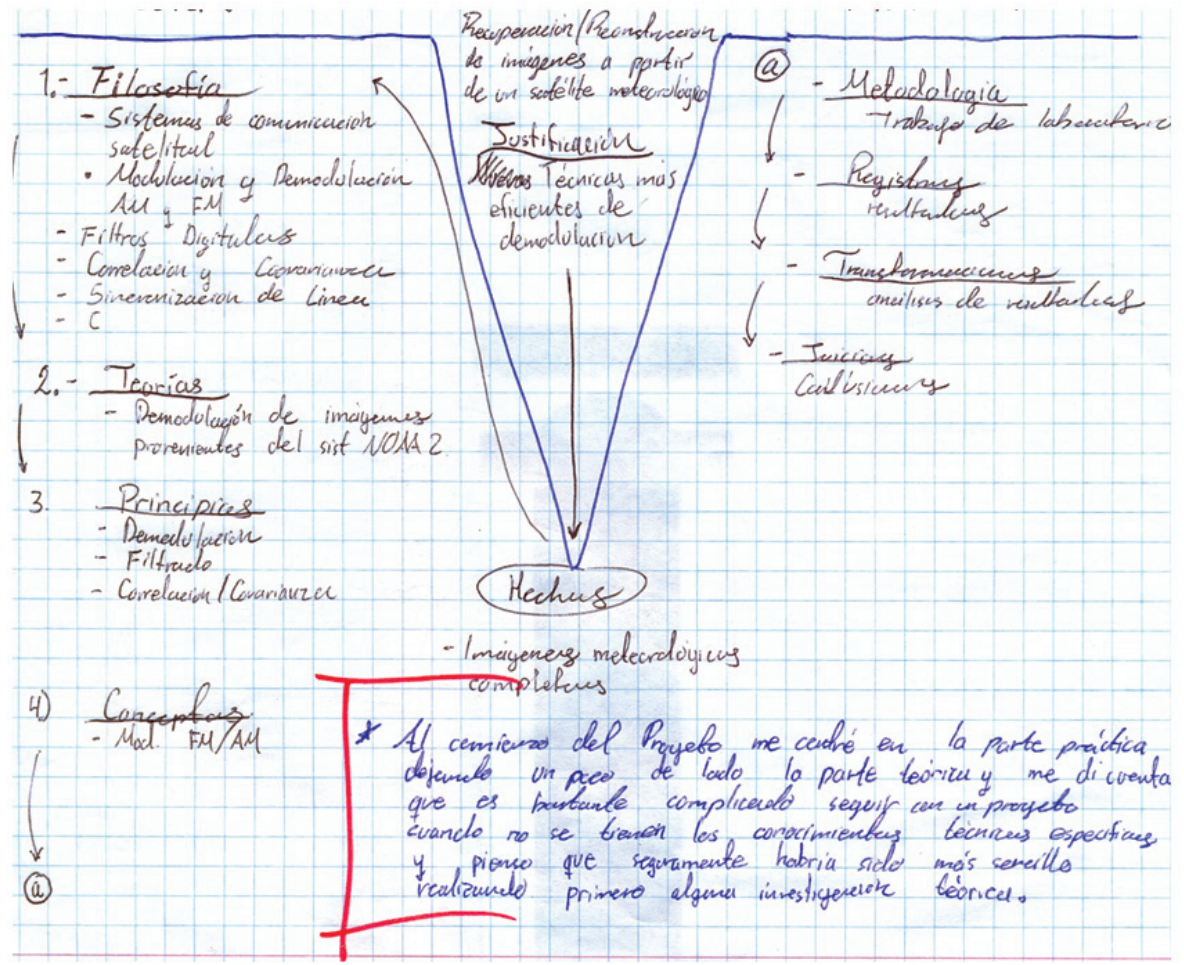

Figura 9. V de Gowin. Elaborada por alumno 3.

En la Figura 10, realizada de forma colaborativa por el grupo 2, la pregunta central y el objetivo se dirigen no hacia los experimentos, sino a desarrollar el planteamiento del problema, incidiendo de nuevo al inicio de la parte teórica en la justificación del mismo. El grupo aunque no hace una diferencia clara entre filosofía, teorías y principios, introduce el estado del arte y lo apoya en los conceptos que guían la búsqueda de bibliografía. La instrucción y posterior elaboración de la $V$, tal y como se puntualiza en la reflexión, les permite integrar la parte teórica y práctica. 
Empleo de herramientas metacognitivas para realizar el proyecto final de licenciatura en la Escuela de Ingeniería ESIME-Culhuacán, del Instituto Politécnico Nacional de México

Ana María Mendioroz Lacambra

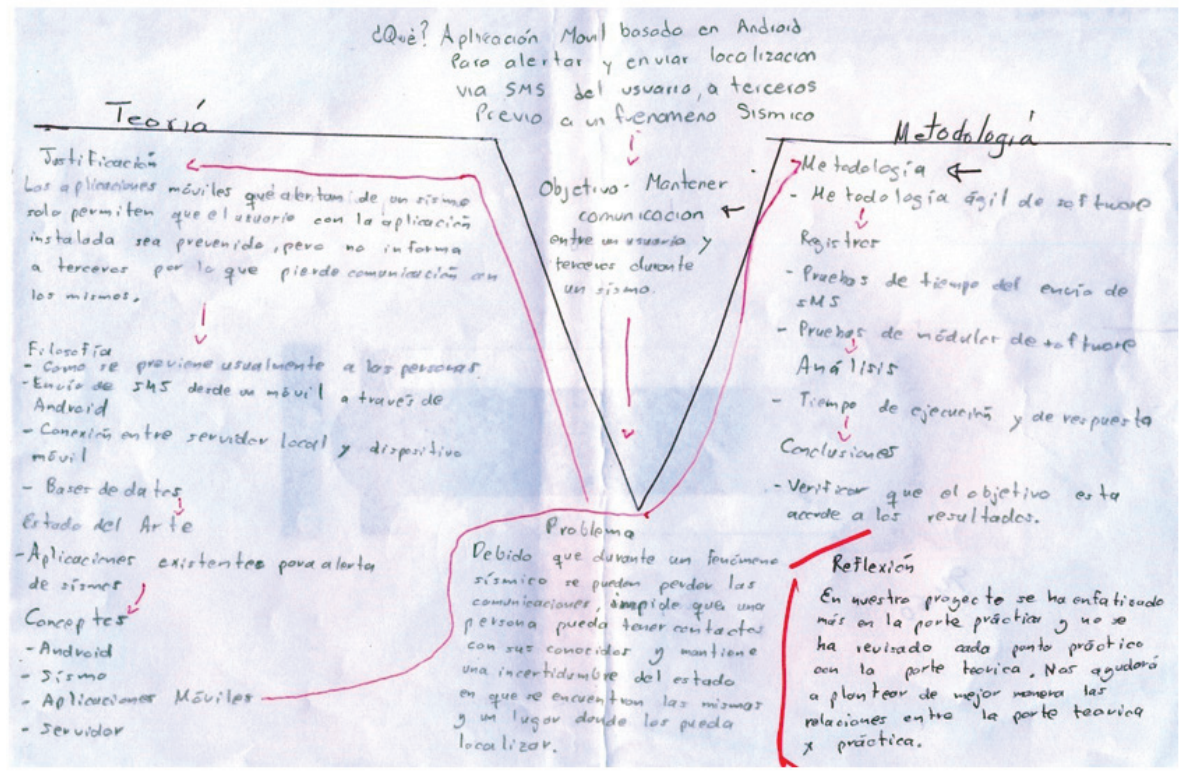

Figura 10. V de Gowin. Elaborada por grupo 2.

En la Figura 11, realizada por el alumno 4, al igual que en la anterior, la pregunta central y el objetivo de la investigación se dirigen a justificar el problema, que vuelve a ser referenciado al inicio de la parte teórica. En este caso, el alumno es capaz de diferenciar los ámbitos que constituyen la parte teórica, adaptándolos al contexto de su proyecto, desde la ejemplificación aportada por la profesora. Los experimentos son introducidos en los registros, identificándolos con los resultados obtenidos; no obstante, al diferenciarlos de las transformaciones, se deduce que el estudiante es consciente de la necesidad de analizar los datos en bruto. La reflexión que realiza, así como la direccionalidad de las flechas, indican que ha sido capaz de integrar los aspectos teóricos y los prácticos, comprendiendo la convergencia entre ambos.

El empleo de la $V$ ha desarrollado en todos los casos analizados, estrategias metacognitivas que han contribuido a visibilizar el proceso de construcción de conocimiento, en tareas de investigación. 


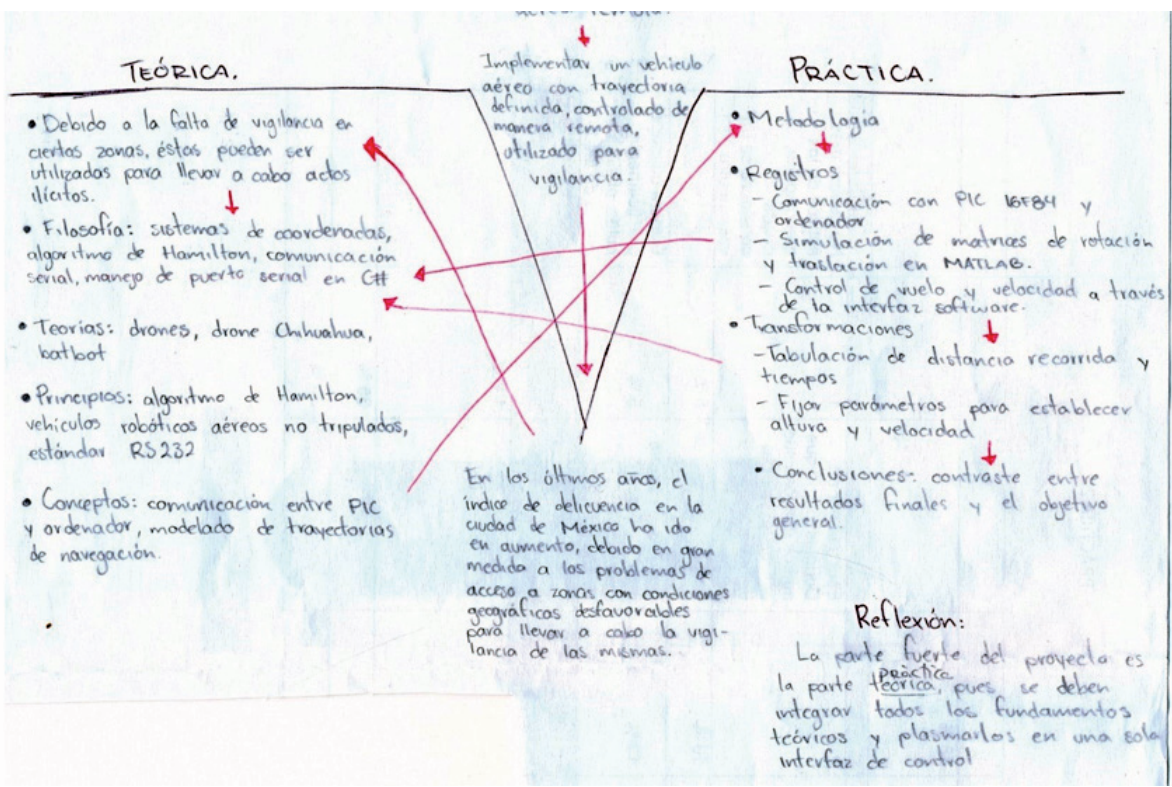

Figura 11. V de Gowin. Elaborada por alumno 4.

\section{Conclusiones}

Tras analizar las evidencias recuperadas sobre el MC, observamos que muchas de las ideas de los alumnos recuperadas en este estudio acerca de su elaboración y empleo, están en la línea de otros precedentes, en los que también se constata la idoneidad del recurso para realizar síntesis y exposiciones, debido a que ayuda a relacionar conceptos y a aprender (Cabral da Costa, 201; Giménez y otros 2009; Manzano, Aguilar Tamayo, Sánchez y Alvarado, 2010; Marques, Moreira, y Cabral da Costa, 2011; Pontes, Serrano, Muñoz, y López, 2011; Pontes, 2012, 2014; Pontes, Serrano, y Muñoz, 2015; Schaal, 2010; Zak y Munson, 2008).

En cuanto al empleo de la $\mathrm{V}$ de Gowin, y a tenor de los resultados obtenidos que apuntan en la misma línea de estudios precedentes, los estudiantes valoran el recurso no sólo como guía para la investigación, al facilitar la visualización de la convergencia entre el conocimiento teórico y el experimental, sino también para constatar la construcción del conocimiento (Guardián, 2009; Guardián, Veloz, Rodríguez y Veloz, 2013; Mendioroz y Guardián, 2014; Virla, 2002).

Para afrontar eficazmente las tareas de investigación, el alumnado debe 
Empleo de herramientas metacognitivas para realizar el proyecto final de licenciatura en la Escuela de Ingeniería ESIME-Culhuacán, del Instituto Politécnico Nacional de México

ana María Mendioroz Lacambra

desarrollar competencias metacognitivas, ya que como indican estudios anteriores (Andrés, 2011), los estudiantes presentan dificultades para visibilizar el proceso de construcción de conocimiento y comunicar eficazmente los resultados. El empleo de herramientas facilitadoras del metaaprendizaje y del metaconocimiento, orientan la investigación y ayudan a evidenciar la convergencia entre teoría y práctica; en suma, facilitan la adquisición de conocimiento de forma autónoma, crítica y autorregulada, proporcionando al alumnado un alto sentimiento de autoeficacia.

\section{Referencias bibliográficas}

Andrés, M. (2011). Modelo Didáctico para Docentes de Ciencias Básicas. Caracas: Fondo Editorial Ipasme.

Andrés, M., Meneses, J. y Pesa, M. (2007). Efectividad meta cognitiva de la heurística V de Gowin en trabajos de laboratorio centrados en la resolución de situaciones problemáticas. Indivisa: Boletín de Estudios e Investigación, 38 (82), 203-216.

Andrés, M., Pesa, M. y Moreira, M. (2006). El trabajo de laboratorio en cursos de física desde la teoría de campos conceptuales. Ciência \& Educação, XXII (2), 129-142.

Banhar, Y. (2007). Developing Mathematical Thinking in Singapore Elementary Schools. Singapore: National Instituted Education.

Belmonte, M. (2004). Mapas Conceptuales y UVES heurísticas de Gowin. Técnicas para todas las áreas de las enseñanzas medias. Madrid: Ediciones Mensajero.

Bruning, R.H., Schraw, G.J., Norby, M. y Ronning, R.R. (2005). Psicología cognitiva y de la instrucción. Madrid: Pearson Prentice Hall.

Cañas, A. y Novak, J. (2014). ¿Qué es un mapa conceptual? Cuadernos de Pedagogía, 488, pp. 54-57.

Cohen, L. y Manion, L. (2002). Métodos de investigación cualitativa. Madrid: Editorial La Muralla, S.A.

Flores, J., Cabarreo, C. y Moreira, M. (2011). Construcción de un marco teórico/conceptual para abordar el trabajo de laboratorio usando el diagrama V: un estudio de caso de la UPEL/IPC. Revista de Investigación 35(73), 241,266.

García Sastre, P., Insausti, M.J. y Merino, M. (2003). Evaluación de los trabajos prácticos mediante diagramas V. Revista Electrónica de Enseñanza de las Ciencias, 2(1), 1-14.

Giménez, V., Rico, J.R., De Alfonseti, N., Lillo, A., Lorenzo, J., Mira-Perceval, T. (2009). El mapa concepual desde la perspectiva del estudiante en los estudios de Trabajo Social. En J.D. Álvarez, N. Pellín y M.T. Tortosa (coords.), La calidad del proceso de enseñanza/aprendizaje universitario desde la perspectiva del cambio (93-101). Barcelona: Universidad de Barcelona

González, F. M. (1992). Los mapas conceptuales de J.D. Novak como instrumentos para la investigación en Didáctica de las Ciencias Experimentales. Enseñanza de las Ciencias, 10, 148-158. 
Empleo de herramientas metacognitivas para realizar el proyecto final de licenciatura en la Escuela de Ingeniería ESIME-Culhuacán, del Instituto Politécnico Nacional de

México

Ana María Mendioroz Lacambra

González, F. M. (2008). El Mapa Conceptual y el Diagrama V. Recursos para la Enseñanza del siglo XXI. Madrid: Narcea.

Graesser, A., Older, B., Pomeroy, P. Witten, S., Lu, S. y Craig, S. (2005). Inferences and questions in science text comprehension. Tarbiya, 36, 103-129.

Gowin, D.B. (1981). Educating. Ithaca. NY: Cornell University Press.

Gowin, D. B. y Álvarez, M.C. (2005). The art of educating with V diagrams. Cambridge: Cambridge University Press.

Guardián, B. D. (2009). Estrategias para promover el aprendizaje significativo de la asignatura de Análisis de Algoritmos en el nivel de Educación Superior. México D.F: UAM-X, Departament of Education.

Guardián, B., Veloz, J., Rodríguez, I. y Veloz, L. (2013). Experiencias de éxito en la aplicación de MC en la carrera de ingeniería en computación. JETT, 4 (1), 73-84.

Higuita-López, D., Molano-Velandia, J. y Rodríguez-Merchán, M. (2011). Competencias necesarias en los grupos de investigación de la Universidad nacional de Colombia que generan desarrollos de base tecnológica. Innovar, 21(41), 209-224.

Hinton, R. (2005). Report of the third Lifelong Learning Network Meeting. Wako-Shi Saitana. Japón: Learning Sciences and Brain Research.

Hipkins, R. (2006). The Nature of the competencies, Concil for Educational Reserches, New Zelanda: Wellington.

Lin, X., Schwartz, D. y Hatano, G. (2005). Toward Teachers Adaptive Metacognition. Educational. Psychologist, 40 (4), 245-255.

Manzano, J., Aguilar, M. F., Sánchez, M. M. y Alvarado, I. (2010). ¡Yo no quiero hacer mapas conceptuales! Estrategias de resistencia de los alumnos universitarios en la apropiación de la herramienta. En: J. Sánchez, A. J. Cañas y J. D. Novak (Eds.). Concept Maps: Making Learning Meaningful. Proceedings of Fourth International Conference on Concept Mapping (Vol. 2, 259-263). Santiago de Chile: Universidad de Chile.

Marques, A., Moreira, M. y Cabral da Costa, S. (2011). Análisis de las opiniones de alumnos de una asignatura de biomecánica acerca de una experiencia con mapas conceptuales como recurso de aprendizaje y estrategia de evaluación. Revista Chilena de Educación Científica, 10 (1), 39-59.

Mendioroz, A. y González, F.M. (2012). El empleo del Mapa Conceptual para elaborar textos expositivos sobre Historia del Arte. En A. Cañas y A. Novak (eds.), Concept Maps: Theory, Methodology, Technology, Proceedings of the Fifth International Conference on Concept Mapping. (vol. III, 82 - 84). Malta: Institute For Human \& Machine Cognition y Universidad de Malta.

Mendioroz, A. (2012). Empleo de la V de Gowin para desarrollar competencias en el máster de Didáctica: propuesta de implementación del recurso y aplicación en un proyecto de intervención en el aula sobre Renacimiento italiano. En F. R. Durán (coords.), Innovación Metodológica y docente en historia, arte y geografía (10481055), Santiago de Compostela: Universidad de Santiago de Compostela.

Mendioroz, A. y Guardián, B. (2014). El empleo de la V de Gowin para responder a las necesidades educativas del alumnado con altas capacidades en Educación Superior, en el área de computación. REDU. Revista de docencia universitaria, 12(4), 457-473. 
Empleo de herramientas metacognitivas para realizar el proyecto final de licenciatura en la Escuela de Ingeniería ESIME-Culhuacán, del Instituto Politécnico Nacional de México

Ana María Mendioroz Lacambra

Moreira, M. A. (2005). Aprendizaje Significativo Crítico. Porto Alegre: Universidad Federal de Porto Alegre.

Moreira, M.A. (2006). Mapas conceituais \& diagramas V. Porto Alegre: Editorial do Autor.

Morales E. (1998). Efecto de una didáctica centrada en la resolución de problemas empleando la técnica heurística $V$ de Gowin y mapas conceptuales en el razonamiento matemático de los alumnos de 9o. grado de educación básica. Revista Latinoamericana de Investigación en Matemática Educativa, 2 (1), 71-84.

Morales, S. y Sánchez, J. (2012). Una experiencia docente sobre aprender a investigar en Educación Social: diseño y desarrollo de proyectos de investigación socioeducativa. Enseñanza \& Teaching, 30, (1), 181-204.

Muñoz, J, Quintero, J. y Munévar, R. (2001). Cómo desarrollar competencias investigativas en educación. Bogotá: Cooperativa Editorial Magisterio.

Novak, J.D. y Gowin D. (2005). Aprendizaje significativo: Técnicas y aplicaciones. USA: Ediciones Pedagógicas/CINCEL.

Plata, M.E. (2011). Procesos de indagación a partir de la pregunta. Una experiencia de formación en investigación. Praxis y Saber 2 (3), 139-172.

Pontes, A., Serrano, R., Muñoz, J. M. y López, I. (2011). Innovación educativa sobre aprendizaje colaborativo con Cmap Tools en la formación inicial docente. Revista Iberoamericana de Evaluación Educativa, 4(2), 136-154.

Pontes, A. (2012). Representación y comunicación del conocimiento con mapas conceptuales en la formación del profesorado de ciencia y tecnología. Revista Eureka sobre Enseñanza y Divulgación de las Ciencias, 9(1), 108-125.

Pontes, A. (2014). Representación del conocimiento físico del alumnado universitario mediante mapas conceptuales elaborados con CmapTools. Alambique: Didáctica de las Ciencias Experimentales, 76, 34-42.

Pontes, A., Serrano, R. y Muñoz, J.M. (2015). Los mapas conceptuales como recurso de interés para la formación inicial del profesorado de enseñanza secundaria: opiniones del alumnado de ciencias sociales y humanidades. Educacion XXI, 18(1), 99-124.

Prytula, M. (2008). Sholarship epistemology an exploratory studys of teacher metacognition within the context of successful learning comunites. Saskatoon, Canadá: University of Saskatchewan.

Reitano, P. y Green, N.C. (2013). Beginning teachers conceptual inderstandings of effective history teaching: examining the change from subject knowers to subject teachers. Asia-Pacif Jornal of Teacher Education, 41 (2), 197-217.

Roncario, N.M. (2012). Revisión sistemática acerca de las competencias investigativas en primera infancia. Horizontes Pedagógicos, 14 (1), 119-134.

Schaal S. (2010). Cognitive and motivational effects of digital concept maps in pre-service science teacher training. Procedia Social and Behavioral Sciences, 2, 640-647.

Sáiz, M. C. y Román, J.M. (2010). Programa de desarrollo de habilidades mentalistas en niños pequeños. Madrid: CEPE.

Veloz, E., Veloz, J., Rodríguez, I. y González, F. (2011). Aplicando MC en teoría de autómatas para evaluar aprendizaje significativo. IN. Investigacio i Innovacio Educativa iSsocioeducativa, 3(1), 161-168.

Veloz, E., Veloz, J., Rodríguez, I. y González, F. (2011). Aplicación de MC a ingeniería 
Empleo de herramientas metacognitivas para realizar el proyecto final de licenciatura en la Escuela de Ingeniería ESIME-Culhuacán, del Instituto Politécnico Nacional de

México

Ana María Mendioroz Lacambra

en computación para evaluar aprendizaje significativo. REDU. Revista de docencia universitaria, 10 (3), 459-475.

Virla H. J. (2002). El uso de la V de Gowin y su Impacto sobre la Realización de Prácticas en el Laboratorio de Electricidad. Docencia Universitaria, III (2), 37-69

Vizcaya, T., Asuaje, R. y Gutiérrez, O. (2009). El método de proyectos y la V de Gowin como estrategias didácticas para el aprendizaje de la Química. Educare, 13 (2), 112137.

Whitebread, D. Coltman, P. Pino, D. Sangster, C. Grau, V. Bingham, S. “..." y Demetriu, D. (2009). The development of two observational tools for assessing metacognition and self-regulated learning in young children. Metacognitive Learning, 4, 63-85

Zak K. M. y Munson B. H. (2008). An exploratory study of elementary preservice teachers' understanding of ecology using concept maps. Journal of Environmental Education, 39(3), 32-46. 
